\title{
Interval Methods for Nonlinear Equation Solving Applications
}

\author{
C. Ryan Gwaltney, Youdong Lin, Luke D. Simoni
}

and Mark A. Stadtherr*

\author{
Department of Chemical and Biomolecular Engineering \\ University of Notre Dame \\ Notre Dame, IN 46556, USA
}

\begin{abstract}
Interval analysis provides techniques that make it possible to determine all solutions to a nonlinear algebraic equation system and to do so with mathematical and computational certainty. Such methods are based on the processing of granules in the form of intervals and thus can be regarded as one facet of granular computing. We review here some of the key concepts used in these methods and then focus on some specific application areas, namely ecological modeling, transition state analysis, and the modeling of phase equilibrium.
\end{abstract}

\section{Overview}

A problem encountered frequently in virtually any field of science, engineering, or applied mathematics is the solution of systems of nonlinear algebraic equations.

\footnotetext{
${ }^{*}$ Author to whom all correspondence should be addressed. Phone: (574) 631-9318; Fax: (574)
} 631-8366; E-mail: markst@nd.edu 
There are many applications in which such systems may have multiple solutions, a single solution, or no solution, with the number of solutions often unknown a priori. Can all solutions be found? If there are no solutions, can this be verified? These are questions that are difficult or impossible to answer using conventional local methods for equation solving. However, methods based on interval analysis are available that can answer these questions, and do so with mathematical and computational rigor. Such methods are based on the processing of granules in the form of intervals and thus can be regarded as one facet of granular computing [6]. The remainder of this chapter is organized as follows: In the next section, a brief summary of interval arithmetic is provided, and some of the key concepts used in interval methods for equation solving are reviewed. In subsequent sections, we focus on specific application areas, namely the modeling of phase equilibrium (Section 3), transition state analysis (Section 4), and ecological modeling (Section 5).

\section{Background}

\subsection{Interval Arithmetic}

Interval arithmetic in its modern form was introduced by Moore [63] and is based on arithmetic conducted on closed sets of real numbers. A real interval $X$ is defined as the set of real numbers between (and including) given upper and lower bounds.

That is, $X=[\underline{X}, \bar{X}]=\{x \in \mathbb{R} \mid \underline{X} \leq x \leq \bar{X}\}$. Here an underline is used to 
indicate the lower bound of an interval, while an overline is used to indicate the upper bound. Unless indicated otherwise, uppercase quantities are intervals, and lower case quantities or uppercase quantities with an underline or overline are real numbers. An interval vector $\mathbf{X}=\left(X_{1}, X_{2}, \ldots, X_{n}\right)^{T}$ has $n$ interval components and can be interpreted geometrically as an $n$-dimensional rectangular convex polytope or "box." Similarly, an $n \times m$ interval matrix $A$ has interval elements $A_{i j}, i=1,2, \ldots, n$ and $j=1,2, \ldots, m$.

Interval arithmetic is an extension of real arithmetic. For a real arithmetic operation op $\in\{+,-, \times, \div\}$, the corresponding interval operation on intervals $X$ and $Y$ is defined by:

$$
X \text { op } Y=\{x \text { op } y \mid x \in X, y \in Y\}
$$

That is, the result of an interval arithmetic operation on $X$ and $Y$ is an interval enclosing the range of results obtainable by performing the operation with any number in $X$ and any number in $Y$. Interval extensions of the elementary functions (sin, cos, exp, log, etc.) can be defined similarly, and computed using interval arithmetic operations on the appropriate series expansions. For dealing with exceptions, such as division by an interval containing zero, extended models for interval arithmetic are available, often based on the extended real system $\mathbb{R}^{*}=\mathbb{R} \cup\{-\infty,+\infty\}$. The concept of containment sets (csets) provides a valuable framework for constructing models for interval arithmetic with consistent handling of exceptions [24, 71]. When machine 
computations using intervals are performed, rounding errors must be handled correctly in order to insure that the result is a rigorous enclosure. Since computers can only represent a finite set of real numbers (machine numbers), the results of floatingpoint arithmetic operations that compute the endpoints of an interval must be determined using a directed (outward) rounding, instead of the standard round-to-nearest procedure. Through the use of interval arithmetic with directed outward rounding, as opposed to floating-point arithmetic, any potential rounding error problems are avoided. Several good introductions to interval analysis, including interval arithmetic and other aspects of computing with intervals, are available [24, 34, 39, 40, 67]. Implementations of interval arithmetic and elementary functions are readily available for a variety of programming environments, including INTLIB [42, 43] for Fortran 77, INTERVAL_ARITHMETIC [38] for Fortran 90, PROFIL/BIAS [70] and FILIB++ [50] for C++, and INTLAB [75] for Matlab. Recent compilers from Sun Microsystems provide direct support for interval arithmetic and an interval data type.

For an arbitrary function $f(x)$, the interval extension, denoted by $F(X)$, encloses all possible values of $f(x)$ for $x \in X$. That is, $F(X) \supseteq\{f(x) \mid x \in X\}$ encloses the range of $f(x)$ over $X$. It is often computed by substituting the given interval $X$ into the function $f(x)$ and then evaluating the function using interval arithmetic. This "natural" interval extension may be wider than the actual range of function values, although it always includes the actual range. The potential overestimation of the function range is due to the "dependency" problem of interval arithmetic, which 
may arise when a variable occurs more than once in a function expression. While a variable may take on any value within its interval, it must take on the same value each time it occurs in an expression. However, this type of dependency is not recognized when the natural interval extension is computed. In effect, when the natural interval extension is used, the range computed for the function is the range that would occur if each instance of a particular variable were allowed to take on a different value in its interval range. For the case in which $f(x)$ is a single-use expression, that is, an expression in which each variable occurs only once, the use of interval arithmetic will always yield the true function range, not an overestimation. For cases in which obtaining a single-use expression is not possible, there are several other approaches that can be used to tighten interval extensions $[24,34,40,67,72]$, including the use of monotonicity [21, 66] and the use of Taylor models [58, 68].

\subsection{Equation Solving Techniques}

There are a many ways intervals may be used in nonlinear equation solving. No attempt is made to survey systematically all such methods here. Instead, we highlight some of the key concepts used in many interval methods for nonlinear equation solving. Many of these concepts can be described in terms of contraction operators, or contractors [34]. Contractors may either reduce the size of, or completely eliminate, the region in which solutions to the equation system of interest are being sought. Consider the nonlinear equation solving problem $\mathbf{f}(\mathbf{x})=\mathbf{0}$, for which real roots are 
sought in an initial interval $\mathbf{X}^{(0)}$. Interval-based strategies exist for contracting, eliminating, or dividing $\mathbf{X}^{(0)}$. Reliable methods for locating all solutions to an equation system are formed by combining these strategies. For a comprehensive treatment of these techniques, several sources are available, including monographs by Neumaier [67], Kearfott [40], Jaulin et al. [34], and Hansen and Walster [24].

\subsubsection{Function range testing}

Consider a search for solutions of $\mathbf{f}(\mathbf{x})=\mathbf{0}$ in an interval $\mathbf{X}$. If an interval extension of $\mathbf{f}(\mathbf{x})$ over $\mathbf{X}$ does not contain zero, that is $\mathbf{0} \notin \mathbf{F}(\mathbf{X})$, then the range of $\mathbf{f}(\mathbf{x})$ over $\mathbf{X}$ does not contain zero, and it is not possible for $\mathbf{X}$ to contain a solution of $\mathbf{f}(\mathbf{x})=\mathbf{0}$. Thus, $\mathbf{X}$ can be eliminated from the search space. The use of interval extensions for function range testing is one simple way an interval can be eliminated as not containing any roots. This is commonly used in nonlinear equation solving methods prior to use of the contraction methods discussed below. A method that makes more extensive use of function range testing was developed by Yamamura [91], based on use

of linear combinations of the component functions of $\mathbf{f}(\mathbf{x})$. An approach for forming the linear combinations based on the inverse of the midpoint of the interval extension of the Jacobian of $\mathbf{f}(\mathbf{x})$ was shown to be very effective.

\subsubsection{Constraint propagation}

Most constraint propagation strategies for nonlinear equation solving are based on the concepts of hull consistency, box consistency, or some combination or variation 
thereof. These are strategies for contracting (shrinking) intervals in which roots are sought, or possibly eliminating them entirely.

The hull consistency form of constraint propagation is based on an interval extension of fixed-point iteration $[24,41]$. Consider a single equation and variable $f(x)=0$, and let it be reformulated into the fixed-point form $x=g(x)$. If $X$ is the search interval, then any roots of $f(x)=0$ must be in the interval $\tilde{X}=G(X)$. It may be possible to shrink the search interval by taking the intersection of $X$ and $\tilde{X}$, that is, $X \leftarrow X \cap \tilde{X}$. If this results in a contraction of $X$, then the process may be repeated. Furthermore, if $X \cap \tilde{X}=\emptyset$, then the current search interval can be eliminated entirely as containing no solutions. If there are different ways to obtain the function $g(x)$, then the process can be repeated using these alternative fixed-point forms. For systems of equations, hull consistency can be applied one equation at a time and one variable at a time (holding other variables constant at their interval values in the current search space). In this way, contractions in one component of the search space can be propagated readily to other components. Clearly, there are many possible strategies for organizing this process.

Another type of constraint propagation strategy is known as box consistency $[24,34]$. In this case, all but one of the variables in an equation system are set to their interval values in the current search space. Now there are one or more equations involving only the remaining variable, say $x_{j}$. These constraints can be used to contract $X_{j}$, the current search range for $x_{j}$. There are various ways to do this, 
including univariate interval-Newton iteration [26] and methods [24] for direct calculation of new bounds for $x_{j}$. This procedure can be repeated using a combination of any equation and any variable in the equation system. Again, this provides a way for contractions in one component of the search space to be propagated to other components. Box consistency and hull consistency tests can also be easily combined [16, 24]. A variety of software packages are available that apply constraint propagation techniques, often in combination with other interval-based methods, to solve systems of equations. These include RealPaver [17], Numerica [86], and ICOS [32].

\subsubsection{Krawczyk and interval-Newton}

The Krawczyk and interval-Newton methods are contraction strategies that have been widely used in the solution of nonlinear equation systems. They also provide a test for the existence of a unique solution in a given interval. Both are generally applied in connection with some bisection or other tessellation scheme [40], thus resulting in a sequence of subintervals to be tested. Let $\mathbf{X}^{(k)}$ indicate an interval in this sequence. Using Krawczyk or interval-Newton, it is possible to contract $\mathbf{X}^{(k)}$, or even eliminate it, and also to determine if a unique solution to $\mathbf{f}(\mathbf{x})=\mathbf{0}$ exists in $\mathbf{X}^{(k)}$

In the Krawczyk method, the interval $\mathbf{K}^{(k)}$ is computed from

$$
\mathbf{K}^{(k)}=\mathbf{K}\left(\mathbf{X}^{(k)}, \mathbf{x}^{(k)}\right)=\mathbf{x}^{(k)}-Y^{(k)} \mathbf{f}\left(\mathbf{x}^{(k)}\right)+\left(I-Y^{(k)} F^{\prime}\left(\mathbf{X}^{(k)}\right)\right)\left(\mathbf{X}^{(k)}-\mathbf{x}^{(k)}\right) .
$$

Here $F^{\prime}\left(\mathbf{X}^{(k)}\right)$ indicates an interval extension of the Jacobian matrix of $\mathbf{f}(\mathbf{x})$, but 
could be any Lipschitz matrix. Also, $\mathbf{x}^{(k)}$ is an arbitrary point in $\mathbf{X}^{(k)}$, and $Y^{(k)}$ is a real preconditioning matrix. The properties of this method have been widely studied $[3,24,34,40,64,65,67]$. Any roots of $\mathbf{f}(\mathbf{x})=\mathbf{0}$ in $\mathbf{X}^{(k)}$ will also be in $\mathbf{K}^{(k)}$, thus giving the contraction scheme $\mathbf{X}^{(k+1)}=\mathbf{X}^{(k)} \cap \mathbf{K}^{(k)}$. It follows that if $\mathbf{X}^{(k)} \cap \mathbf{K}^{(k)}=\emptyset$, then $\mathbf{X}^{(k)}$ contains no roots and can be eliminated. An additional property is that if $\mathbf{K}^{(k)}$ is in the interior of $\mathbf{X}^{(k)}$, then there is a unique root in $\mathbf{X}^{(k)}$. If $\mathbf{X}^{(k)}$ cannot be eliminated or sufficiently contracted, or cannot be shown to contain a unique root, then it is bisected, and the procedure repeated on each resulting interval. Several improvements to the basic Krawczyk method have been suggested, including a bicentered method [79] a boundary-based method [79, 80], and a componentwise version of the algorithm [61].

In the interval-Newton method, the interval $\mathbf{N}^{(k)}=\mathbf{N}\left(\mathbf{X}^{(k)}, \mathbf{x}^{(k)}\right)$ is determined from the linear interval equation system

$$
Y^{(k)} F^{\prime}\left(\mathbf{X}^{(k)}\right)\left(\mathbf{N}^{(k)}-\mathbf{x}^{(k)}\right)=-Y^{(k)} \mathbf{f}\left(\mathbf{x}^{(k)}\right) .
$$

This method has also been widely studied $[4,9,24,34,40,67]$ and has properties similar to the Krawczyk method. Any roots in $\mathbf{X}^{(k)}$ are also in $\mathbf{N}^{(k)}$, so the contraction $\mathbf{X}^{(k+1)}=\mathbf{X}^{(k)} \cap \mathbf{N}^{(k)}$ can be used. If $\mathbf{X}^{(k)} \cap \mathbf{N}^{(k)}=\emptyset$, then $\mathbf{X}^{(k)}$ can be eliminated. Furthermore, if $\mathbf{N}^{(k)}$ is in the interior of $\mathbf{X}^{(k)}$, there is a unique root in $\mathbf{X}^{(k)}$. In this case, the interval-Newton procedure can be repeated and converges quadratically to a narrow enclosure of the root. Alternatively, an approximation of the root can be found using a standard point-Newton algorithm starting from any point in $\mathbf{X}^{(k)}$. Again, if 
$\mathbf{X}^{(k)}$ cannot be eliminated or sufficiently shrunk, or cannot be shown to contain a unique root, it is bisected. $\mathbf{N}^{(k)}$ can be obtained from the linear interval equation system (3) in various ways. However, an interval Gauss-Seidel procedure [22] is widely used. In this case, $\mathbf{N}^{(k)}$ is never obtained explicitly, since after each component $N_{i}^{(k)}$ is computed, it is intersected with $X_{i}^{(k)}$, and the result is then used in computing subsequent components of $\mathbf{N}^{(k)}$. For a fixed preconditioning matrix, the enclosure provided by the interval-Newton method using Gauss-Seidel is at least as good as that provided by the Krawczyk method $[22,67]$. Nevertheless, the Krawczyk method appears attractive, because it is not necessary to bound the solution of a system of linear interval equations. However, in practice the interval Gauss-Seidel procedure is a very simple and effective way to deal with the linear equation system. Overall, interval-Newton with Gauss-Seidel is regarded as computationally more efficient than the Krawczyk method [23, 24].

There are many variations on the interval-Newton method, corresponding to different choices of the real point $\mathbf{x}^{(k)}$ and preconditioning matrix $Y^{(k)}$, different strategies for choosing the bisection coordinate, and different ways to bound the solution of Eq. (3). The real point $\mathbf{x}^{(k)}$ is typically taken to be the midpoint of $\mathbf{X}^{(k)}$, and the preconditioning matrix $Y^{(k)}$ is often taken to be either the inverse of the midpoint of $F^{\prime}\left(\mathbf{X}^{(k)}\right)$, or the inverse of the Jacobian evaluated at the midpoint of $\mathbf{X}^{(k)}$. However, these choices are not necessarily optimal [37]. For example, several alternative preconditioning strategies are given by Kearfott et al. [44]. Gau and Stadtherr [14] combined 
one of these methods, a pivoting preconditioner, with a standard inverse midpoint scheme, and was able to obtain significant performance gains compared to the use of the inverse midpoint preconditioner alone. For the choice of the real point $\mathbf{x}^{(k)}$, one alternative strategy is to use an approximation to a root of the equation system, perhaps obtained using a local equation solver. Gau and Stadtherr [14] suggested a real-point selection scheme that seeks to minimize the width of the intersection between $X_{i}^{(k)}$ and $N_{i}^{(k)}$. Several bisection or other box splitting strategies have been studied [24, 40, 45, 65]. The maximum smear heuristic [45], in which bisection is done on the coordinate whose range corresponds to the maximum width in the function range, is often, but not always, an effective choice. For bounding the solution of Eq. (3) there are many possible approaches, though, as noted above, the preconditioned interval Gauss-Seidel approach is typically quite effective. One alternative, described by Lin and Stadtherr $[51,53]$, uses a linear programming strategy along with a realpoint selection scheme to provide sharp enclosures of the solution $\mathbf{N}^{(k)}$ to Eq. (3). Though, in general, sharply bounding the solution set of a linear interval equation system is NP-hard, for the special case of interval-Newton, this linear programming approach can efficiently provide exact (within roundout) bounds. Finally, it should be noted that a slope matrix can be used in Eqs. (2-3) instead of a Lipschitz matrix. In this case, the test for enclosure of a unique root is no longer applicable, unless some type of compound algorithm is used [74].

In implementing the interval-Newton method, values of $\mathbf{f}(\mathbf{x})$ are computed using 
interval arithmetic to bound rounding errors. Thus, in effect, $\mathbf{f}(\mathbf{x})$ is interval valued. In general, the interval-Newton method can be used to enclose the solution set of any interval-valued function. For example, consider the problem $\mathbf{f}(\mathbf{x}, p)=\mathbf{0}$, where $p$ is some parameter. If the value of $p$ is uncertain but is known to be in the interval $P$, then we have the interval-valued function $\mathbf{F}(\mathbf{x}, P)$ and the problem is to enclose the solution set of $\mathbf{F}(\mathbf{x}, P)=\mathbf{0}$. This solution set is defined by $\mathcal{S}=\{\mathbf{x} \mid \mathbf{f}(\mathbf{x}, p)=$ $\mathbf{0}, p \in P\}$. An interval enclosure of $\mathcal{S}$ can be found readily using the interval-Newton method, though generally, due to bounding of rounding errors, it will not be the smallest possible interval enclosure. However, since $\mathcal{S}$ is often not an interval, even its tightest interval enclosure may still represent a significant overestimation. To more closely approximate $\mathcal{S}$, one can divide $P$ into subintervals, obtain an interval enclosure of the solution set over each subinterval, and then take the union of the results.

Implementation of interval methods for nonlinear equation solving typically employ a combination of one or more of the concepts outlined above $[10,16,41]$, perhaps also in connection with some manipulation of the equation system to be solved $[46,47,91]$. Often function range testing and constraint propagation techniques are used first to contract intervals, as these methods have low computational overhead. Then, more costly interval-Newton steps can be applied to the contracted intervals to obtain final solution enclosures. In most such equation solving algorithms, the intervals can be treated as independent granules of data. Thus, parallel implementa- 
tions of interval methods are generally apparent, though must be done with proper attention to load balancing in order to be most effective [13, 48, 76].

In the subsequent sections, we will look at some specific applications of interval methods for nonlinear equation solving. The core steps in the algorithm used to solve these problems can be outlined as follows: For a given $\left.\mathbf{X}^{(k)}, 1\right)$ Apply function range test; if $\mathbf{X}^{(k)}$ is not eliminated, then 2) Apply hull consistency (this is done on a problem specific basis); if $\mathbf{X}^{(k)}$ is not eliminated, then 3) Apply interval-Newton, using either the hybrid preconditioning technique of Gau and Stadtherr [14], or the linear programming method of Lin and Stadtherr [53]; if $\mathbf{X}^{(k)}$ is not eliminated, or

a unique root in $\mathbf{X}^{(k)}$ not identified, then 4) Bisect $\mathbf{X}^{(k)}$. This is only one possible way to implement an interval method for nonlinear equation solving applications. However, it has proved to be effective on a wide variety of problems, some of which are discussed below. The applications considered next are purely equation solving problems. However, since many optimization problems can be easily converted into an equivalent system of equations, the techniques described above are also often applied to problems requiring global optimization, typically in connection with some branch-and-bound procedure.

\section{Modeling of Liquid-Liquid Phase Equilibrium}

The modeling of phase behavior is a rich source of problems in which interval methods can play an important role, by ensuring that correct results are reliably 
obtained [8, 89]. Of interest is the development and use of models for predicting the number, type (liquid, vapor, solid), and composition of the phases present at equilibrium for mixtures of chemical components at specified conditions. In model development, parameter estimation problems arise, which typically require solution of a nonconvex optimization problem. Unfortunately, it is not uncommon to find that literature values for parameters are actually locally, but not globally optimal [11]. Use of parameters that are not globally optimal may result in rejection of a model that would otherwise be accepted if globally optimal parameters were used. For the case of vapor-liquid equilibrium modeling, Gau and Stadtherr [11, 12] have used an interval method to guarantee that the globally optimal parameters are found. After models are developed, they are used to compute the phase equilibrium for mixtures of interest. This is another global optimization problem, the global minimization of the total Gibbs energy in the case of specified temperature and pressure. Again, it is not uncommon to find literature solutions that are locally optimal only, and thus do not represent stable equilibrium states [89]. For the phase stability and equilibrium problems, and for related phase behavior calculations, there have been a number of successful applications of interval methods to the underlying equation solving and optimization problems [8, 27-31, 55, 56, 60, 78, 82-84, 88-90].

In this section, we will focus on the problem of parameter estimation in the modeling of liquid-liquid equilibrium. This can be formulated as a nonlinear equation solving problem involving only two equations and variables. However, the number of 
solutions to this system is unknown a priori, and it is not uncommon to see incorrect solutions reported in the literature.

\subsection{Problem Formulation}

Consider liquid-liquid equilibrium in a two-component system at fixed temperature and pressure. For this case, the necessary and sufficient condition for equilibrium is that the total Gibbs energy be at a global minimum. The first-order optimality conditions on the Gibbs energy lead to the equal activity conditions,

$$
a_{i}^{\mathrm{I}}=a_{i}^{\mathrm{II}}, \quad i=1,2,
$$

stating the equality of activities of each component (1 and 2) in each phase (I and II). This is a necessary but not sufficient condition for equilibrium. Given an activity coefficient model $\left(a_{i}=\gamma_{i} x_{i}\right)$, expressed in terms of observable component mole fractions $x_{1}$ and $x_{2}=1-x_{1}$, and activity coefficients $\gamma_{1}$ and $\gamma_{2}$ expressed in terms of composition and two binary parameters $\theta_{12}$ and $\theta_{21}$, then the equal activity conditions can be expressed as

$$
x_{i}^{\mathrm{I}} \gamma_{i}^{\mathrm{I}}\left(x_{1}^{\mathrm{I}}, x_{2}^{\mathrm{I}}, \theta_{12}, \theta_{21}\right)=x_{i}^{\mathrm{II}} \gamma_{i}^{\mathrm{II}}\left(x_{1}^{\mathrm{II}}, x_{2}^{\mathrm{II}}, \theta_{12}, \theta_{21}\right), \quad i=1,2
$$

Experimental measurements of the compositions of both phases are available. Thus, in Eq. (5), the values of $x_{1}^{\mathrm{I}}, x_{1}^{\mathrm{II}}, x_{2}^{\mathrm{I}}$, and $x_{2}^{\mathrm{II}}$ are fixed. This results in a system of two equations in the two parameters $\theta_{12}$ and $\theta_{21}$. This provides a widely used approach for parameter estimation in activity coefficient models for liquid-liquid equilibrium 
$[73,81]$, as generally it is possible to use physical grounds to reject all but one solution to Eq. (5). Parameter solutions are generally sought using local methods with multistart. A curve-following approach can also be used [33], but its reliability is stepsize dependent and is not guaranteed. In this section, we will use an interval-Newton approach, as outlined at the end of Section 2, to determine reliably all solutions to Eq. (5) for the case in which the NRTL activity coefficient model is used.

In the NRTL model, the activity coefficients for use in Eq. (5) are given by

$$
\begin{aligned}
& \ln \gamma_{1}=x_{2}^{2}\left[\tau_{21}\left(\frac{G_{21}}{x_{1}+x_{2} G_{21}}\right)^{2}+\frac{\tau_{12} G_{12}}{\left(x_{2}+x_{1} G_{12}\right)^{2}}\right] \\
& \ln \gamma_{2}=x_{1}^{2}\left[\tau_{12}\left(\frac{G_{12}}{x_{2}+x_{1} G_{12}}\right)^{2}+\frac{\tau_{21} G_{21}}{\left(x_{1}+x_{2} G_{21}\right)^{2}}\right],
\end{aligned}
$$

where

$$
\begin{gathered}
\tau_{12}=\frac{g_{12}-g_{22}}{R T}=\frac{\Delta g_{12}}{R T} \\
\tau_{21}=\frac{g_{21}-g_{11}}{R T}=\frac{\Delta g_{21}}{R T} \\
G_{12}=\exp \left(-\alpha_{12} \tau_{12}\right) \\
G_{21}=\exp \left(-\alpha_{21} \tau_{21}\right) .
\end{gathered}
$$

Here $g_{i j}$ is an energy parameter characteristic of the $i-j$ interaction, and the parameter $\alpha=\alpha_{12}=\alpha_{21}$ is related to the nonrandomness in the mixture. The nonrandomness parameter $\alpha$ frequently is taken to be fixed when modeling liquid-liquid equilibrium. The binary parameters that must be determined from experimental data are then $\theta_{12}=\Delta g_{12}$ and $\theta_{21}=\Delta g_{21}$ 


\section{$3.2 n$-Butanol and Water}

Consider a mixture of $n$-butanol (component 1) and water (component 2) at $T=363 \mathrm{~K}$ and atmospheric pressure. Liquid-liquid phase equilibrium is observed experimentally with phase compositions $x_{1}^{I}=0.020150$ and $x_{1}^{I I}=0.35970$. Heidemann and Mandhane [25] modeled this system using NRTL with $\alpha=0.4$. They obtained three solutions for the binary parameters, as shown in Table 1 , in terms of $\tau_{12}$ and $\tau_{21}$. Applying the interval method to solve this system of nonlinear equations, with an initial search interval of $\theta_{12} \in\left[-1 \times 10^{6}, 1 \times 10^{6}\right]$ and $\theta_{21} \in\left[-1 \times 10^{6}, 1 \times 10^{6}\right]$, we find only two solutions, as also shown in Table 1. The extra solution found by Heidemann and Mandhane [25] is well within the search space used by the interval method, so clearly is a spurious solution resulting from numerical difficulties in the local method used to solve Eq. (5). This can be verified by direct substitution of solution 3 into the equal activity conditions. When equal activity is expressed in the form of Eq. (5), the residuals for solution 3 are close to zero. However, when equal activity is expressed in terms of $\ln x_{i}$ and $\ln \gamma_{i}$, by taking the logarithm of both sides of Eq. (5), it becomes clear that the residuals for solution 3 are not really zero.

\subsection{1, 4-Dioxane and 1,2,3-Propanetriol}

Consider a mixture of 1,4-dioxane (component 1$)$ and 1,2,3-propanetriol at $T=$ $298 \mathrm{~K}$ and atmospheric pressure. Liquid-liquid phase equilibrium is observed experimentally with phase compositions $x_{1}^{I}=0.2078$ and $x_{1}^{I I}=0.9934$. Mattelin and 
Verhoeye [59] modeled this system using NRTL with various values of $\alpha$. We will focus on the case of $\alpha=0.15$. They obtained six solutions for the binary parameters, which are reported graphically without giving exact numerical values. Applying the interval method, with the same initial search interval as given above, we find only four solutions, as shown in Table 2 in terms of $\tau_{12}$ and $\tau_{21}$. The extra solutions found by Mattelin and Verhoeye [59] are well within the search space used by the interval method. Again, it appears that numerical difficulties in the use of local methods has led to spurious solutions.

\subsection{Remarks}

In this section, we have seen a small nonlinear equation system that in some cases is numerically difficult to solve using standard local methods, as evident from the reporting of spurious roots in the literature. Using an interval-Newton method, tight enclosures of all roots in the initial search space could be found very easily and efficiently, with computational times on the order of seconds $(3.2 \mathrm{GHz}$ Intel Pentium 4).

\section{Transition State Analysis}

In molecular modeling, the search for features on a potential energy hypersurface is often required and is a very challenging computational problem. In some cases, finding a global minimum is required, but the existence of a very large number of local 
minima, the number of which may increase exponentially with the size of a molecule or the number of molecules, makes the problem extremely difficult. Interval methods can play a role in solving these problems $[49,54]$, but are limited in practice to problems of relatively low dimension. In other problems in computational chemistry, it is desired to find all stationary points. Interval methods for equation solving have been applied to one such problem, involving the use of lattice density functional theory to model adsorption in a nanoscale pore, by Maier and Stadtherr [57]. Another such problem is transition state analysis, as summarized below, and described in more detail by Lin and Stadtherr [52].

Transition state theory is a well-established method which, by providing an approach for computing the kinetics of infrequent events, is useful in the study of numerous physical systems. Of particular interest here is the problem of computing the diffusivity of a sorbate molecule in a zeolite. This can be done using transition state analysis, as described by June et al. [35]. It is assumed that diffusive motion of the sorbate molecules through the zeolite occurs by a series of uncorrelated hops between potential energy minima in the zeolite lattice. A sorption state or site is constructed around each minimum of the potential energy hypersurface. Any such pair of sites $i$ and $j$ then is assumed to be separated by a dividing surface on which a saddle point of the potential energy hypersurface is located. The saddle point can be viewed as the transition state between sites, and a pair of steepest decent paths from the saddle point connect the minima associated with the $i$ and $j$ sites. Obviously, in this appli- 
cation, and in other applications of transition-state theory, finding all local minima and saddle points of the potential energy surface, $\mathcal{V}$, is critical. We show here, using a sorbate-zeolite system, the use of an interval-Newton method, as outlined at the end of Section 2, to find all stationary points of a potential energy surface.

Stationary points satisfy the condition $\mathrm{g}=\nabla \mathcal{V}=\mathbf{0}$; that is, at a stationary point, the gradient of the potential energy surface is zero. Using the eigenvalues of $H=\nabla^{2} \mathcal{V}$, the Hessian of the potential energy surface, stationary points can be classified into local minima, local maxima, and saddle points (of order determined by the number of negative eigenvalues). There are a number of methods for locating stationary points. A Newton or quasi-Newton method, applied to solve the nonlinear equation system $\nabla \mathcal{V}=\mathbf{0}$, yields a solution whenever the initial guess is sufficiently close to a stationary point. This method can be used in an exhaustive search, using many different initial guesses, to locate stationary points. The set of initial guesses to use might be determined by the user (intuitively or arbitrarily) or by some type of stochastic multistart approach. Another popular approach is the use of eigenmodefollowing methods, as done, for example, by Tsai and Jordan [85]. These methods can be regarded as variations of Newton's method. In an eigenmode-following algorithm, the Newton step is modified by shifting some of the eigenvalues of the Hessian (from positive to negative or vice versa). By selection of the shift parameters, one can effectively find the desired type of stationary points, e.g., minima and first-order saddles. There are also a number of other approaches, many involving some stochastic 
component, for finding stationary points.

In the context of sorbate-zeolite systems, June et al. [35] use an approach in which minima and saddle points are located separately. A three step process is employed in an exhaustive search for minima. First, the volume of the search space (one asymmetric unit) is discretized by a grid with a spacing of approximately $0.2 \AA$, and the potential and gradient vector are tabulated on the grid. Second, each cube formed by a set of nearest-neighbor grid nodes is scanned, and the three components of the gradient vector on the eight vertices of the cube are checked for changes in sign. Finally, if all three components are found to change sign on two or more vertices of the cube, a BFGS quasi-Newton minimization search algorithm is initiated to locate a local minimum, using the coordinates of the center of the cube as the initial guess. Two different algorithms are tried for determining the location of saddle points. One searches for global minimizers in the function $\mathbf{g}^{\mathrm{T}} \mathbf{g}$, i.e. the sum of the squares of the components of the gradient vector. The other algorithm, due to Baker [5], searches for saddle points directly from an initial point by maximizing the potential energy along the eigenvector direction associated with the smallest eigenvalue and by minimizing along directions associated with all other eigenvalues of the Hessian.

All the methods discussed above have a major shortcoming. They provide no guarantee that all local minima and saddle points of interest will actually be found. One approach to resolving this difficulty is given by Westerberg and Floudas [87], who transform the equation-solving problem $\nabla \mathcal{V}=\mathbf{0}$ into an equivalent optimization 
problem that has global minimizers corresponding to the solutions of the equation system (i.e., the stationary points of $\mathcal{V}$ ). A deterministic global optimization algorithm, based on a branch-and-bound strategy with convex underestimators, then is used to find these global minimizers. Whether all stationary points are actually found depends on proper choice of a parameter $(\alpha)$ used in obtaining the convex underestimators, and Westerberg and Floudas do not use a method that guarantees a proper choice. However, there do exist techniques $[1,2]$, based on an interval representation of the Hessian, that in principle could be used to guarantee a proper value of $\alpha$, though likely at considerable computational expense. We demonstrate here an approach in which interval analysis is applied directly to the solution of $\nabla \mathcal{V}=\mathbf{0}$ using an interval-Newton methodology. This provides a mathematical and computational guarantee that all stationary points of the potential energy surface are found (or, more precisely, enclosed within an arbitrarily small interval).

\subsection{Problem Formulation}

Zeolites are materials in which $\mathrm{AlO}_{4}$ and $\mathrm{SiO}_{4}$ tetrahedra are the building blocks of a variety of complex porous structures characterized by interconnected cavities and channels of molecular dimensions [36]. Silicalite contains no aluminum and thus no cations. This has made it a common and convenient choice as a model zeolite system. The crystal structure of silicalite, well known from X-ray diffraction studies [69], forms a three-dimensional interconnected pore network through which a sorbate molecule 
can diffuse. In this work, the phase with orthorhombic symmetry is considered, and a rigid lattice model, in which all silicon and oxygen atoms in the zeolite framework are occupying fixed positions and there is perfect crystallinity, is assumed. One spherical sorbate molecule (united atom) will be placed in the lattice, corresponding to infinitely dilute diffusion. The system is comprised of 27 unit cells, each of which is $20.07 \times 19.92 \times 13.42 \AA$ with 96 silicon atoms and 192 oxygen atoms.

All interactions between the sorbate and the oxygen atoms of the lattice are treated atomistically with a truncated Lennard-Jones 6-12 potential. That is, for the interaction between the sorbate and oxygen atom $i$, the potential is given by

$$
\mathcal{V}_{i}= \begin{cases}\frac{a}{r_{i}^{12}}-\frac{b}{r_{i}^{6}} & r_{i}<r_{\text {cut }} \\ 0 & r_{i} \geq r_{\text {cut }}\end{cases}
$$

where $a$ is a repulsion parameter, $b$ is an attraction parameter, $r_{\text {cut }}$ is the cutoff distance, and $r_{i}$ is the distance between the sorbate and oxygen atom $i$. This distance is given by

$$
r_{i}^{2}=\left(x-x_{i}\right)^{2}+\left(y-y_{i}\right)^{2}+\left(z-z_{i}\right)^{2},
$$

where $(x, y, z)$ are the Cartesian coordinates of the sorbate, and $\left(x_{i}, y_{i}, z_{i}\right), i=1, \ldots, N$ are the Cartesian coordinates of the $N$ oxygen atoms. The silicon atoms, being recessed within the $\mathrm{SiO}_{4}$ tetrahedra, are neglected in the potential function. Therefore, the total potential energy, $\mathcal{V}$, of a single sorbate molecule in the absence of neighboring sorbate molecules is represented by a sum over all lattice oxygens,

$$
\mathcal{V}=\sum_{i=1}^{N} \mathcal{V}_{i}
$$


The interval-Newton approach is applied to determine the sorbate locations $(x, y, z)$ that are stationary points on the potential energy surface $\mathcal{V}$ given by Eq. (10), that is, to solve the nonlinear equation system $\nabla \mathcal{V}=\mathbf{0}$. To achieve tighter interval extensions of the potential function and its derivatives, and thus improve the performance of the interval-Newton method, the mathematical properties of the Lennard-Jones potential and its first- and second-order derivatives can be exploited, as described in detail by Lin and Stadtherr [52].

\subsection{Results and Discussion}

Due to the orthorhombic symmetry of the silicalite lattice, the search space for stationary points is only one asymmetric unit, $[0,10.035] \times[0,4.98] \times[0,13.42] \AA$, which is one-eighth of a unit cell. This defines the initial interval for the intervalNewton method, namely $X^{(0)}=[0,10.035] \AA, Y^{(0)}=[0,4.98] \AA$, and $Z^{(0)}=[0,13.42]$ Å. Following June et al. [35], stationary points with extremely high potential, such as $\mathcal{V}>0$, will not be sought. To do this, we calculate the interval extension of $\mathcal{V}$ over the interval currently being tested. If its lower bound is greater than zero, the current interval is discarded.

Using the interval-Newton method, with the linear programming strategy of Lin and Stadtherr [53], a total of 15 stationary points were found in a computation time of 724 seconds (1.7 GHz Intel Xeon). The locations of the stationary points, their energy value, and their type are listed in Table 3. Five local minima were found, 
along with 8 first-order saddle points and two second-order saddle points. June et al. [35] report the same five local minima, as well as 9 of the 10 saddle points. They do not report finding the lower energy second-order saddle point (saddle point \#14 in Table 3).

The second-order saddle point \#14, not reported by June et al. [35], is very close to the first-order saddle point \#13, and slightly lower in energy. Apparently neither of the two methods tried by June et al. [35] was able to locate this point. The first method they tried uses the same grid-based optimization scheme used to locate local minima in $\mathcal{V}$, but instead applied to minimize $\mathbf{g}^{\mathrm{T}} \mathbf{g}$. However, stationary points \#13 and \#14 are approximately $0.1 \AA$ apart, while the grid spacing they used was approximately $0.2 \AA$. This illustrates the danger in using grid-based schemes for finding all solutions to a problem. By using the interval methods described here, one never needs to be concerned about whether a grid spacing is fine enough to find all solutions. The second method they tried was Baker's algorithm [5], as described briefly above, but it is unclear how they initialized the algorithm. A key advantage of the interval method is that no point initialization is required. Only an initial interval must be supplied, here corresponding to one asymmetric unit, and this is determined by the geometry of the zeolite lattice. Thus, in this context, the interval method is initialization independent. 


\subsection{Remarks}

Lin and Stadtherr [53] have also studied two other sorbate-zeolite systems, and used the interval method to find all stationary points on the potential energy surfaces. While we have concentrated here on problems involving transition-state analysis of diffusion in zeolites, we anticipate that the method will be useful in many other types of problems in which transition-state theory is applied.

\section{Food Web Models}

Ecological models, including models of food webs, are increasingly being used as aids in the management and assessment of ecological risks. As a first step in using a food web model, an understanding is needed of the predicted equilibrium states (steady states) and their stability. To determine the equilibrium states, a system of nonlinear equations must be solved, with the number of solutions often not known a priori. Finding bifurcations of equilibria (parameter values at which the number of equilibrium states or their stability changes) is another problem of interest, which can also be formulated as a nonlinear equation solving problem. For both of these problems, continuation methods are typically used, but are initialization dependent and provide no guarantees that all solutions will be found. Gwaltney et al. [20] and Gwaltney and Stadtherr [19] have demonstrated the use of an intervalNewton method to find equilibrium states and their bifurcations for some simple food

chain models. Interval methods have been also successfully applied to the problem 
of locating equilibrium states and singularities in traditional chemical engineering problems, such as reaction and reactive distillation systems $[7,15,62,77]$. We will consider here a seven-species food web, and use an interval-Newton approach, as outlined at the end of Section 2, to solve for all steady states predicted by the model.

\subsection{Problem Formulation}

The seven-species food web is shown schematically in Fig. 1. It involves two producers (species 1 and 2) and five consumers (species 3-7). The producers are assumed to grow logistically, while the consumers obey predator response functions that will be specified below.

The model equations (balance equations) are, for $i=1, \ldots, 7$,

$$
f_{i}(\mathbf{m})=\frac{d m_{i}}{d t}=m_{i} g_{i}(\mathbf{m})=m_{i}\left[r_{i}+\sum_{j=1}^{7} a_{i j} p_{i j}(\mathbf{m})\right]
$$

Here the variables are the species biomasses $m_{i}, i=1, \ldots, 7$, which are the components of the biomass vector $\mathbf{m}$. The constants $a_{i j}$ represent combinations of different model parameters, and also indicate the structure of the food web. The constants $r_{i}$ consist of intrinsic growth and death rate parameters. The functions $p_{i j}(\mathbf{m})$ are determined by the choice of predator response function for the predator-prey interaction involving species $i$ and $j$. For the 1-3 interaction, we assume a hyperbolic response function (Holling type II). This leads to $p_{13}(\mathbf{m})=m_{3} /\left(m_{1}+B_{13}\right)$, and $p_{31}(\mathbf{m})=m_{1} /\left(m_{1}+B_{13}\right)$, where $B_{13}$ is the half-saturation constant for consumption of species 1 by species 3. For all other interactions, we assume a linear response 
function (Lotka-Volterra), giving $p_{i j}(\mathbf{m})=m_{j}$. Values of all constants in the model are given by Gwaltney [18]. To determine the equilibrium states predicted by this model, solution of the nonlinear equation system

$$
f_{i}(\mathbf{m})=m_{i} g_{i}(\mathbf{m})=0, \quad i=1, \ldots, 7
$$

is required.

\subsection{Results and Discussion}

There are two basic strategies for solving the equation system. In the simultaneous strategy, we simply solve Eq. (12) directly, as a system of seven equations in seven variables. In the sequential strategy, a sequence of smaller problems is solved, one for each feasible zero-nonzero state. A set of feasible zero-nonzero states can be constructed from the structure of the food web. For example, the state [1030060] (this indicates that species 1, 3 and 6 have nonzero biomasses, and that species 2 , 4, 5 and 7 are absent) is feasible. However, the state [1204067] is not feasible, since in the absence of species 3 and 5 species 6 cannot exist. For a relatively small food web, it is not difficult to construct the set of feasible zero-nonzero states. However, for large food webs this is nontrivial, as the number of such states can become very large. For the seven-species web of interest here, there are 55 feasible zero-nonzero states. For each zero-nonzero state, an equation system is formulated to solve for the corresponding steady states. For example, for the $[1030060]$ state, $m_{1} \neq 0$, thus it is required that $g_{1}=0$. Similarly, $g_{3}=0$ and $g_{6}=0$. This provides three equations in 
the three nonzero variables $m_{1}, m_{3}$, and $m_{6}$. The remaining components of Eq. (12) are satisfied because $m_{2}=m_{4}=m_{5}=m_{7}=0$.

An interval-Newton approach was used to solve the nonlinear equation system (12) in connection with both the simultaneous and sequential approaches. This was done for several different values of the model parameter $K_{2}$, the carrying capacity for producer species 2. A partial set of results $\left(m_{1}\right.$ and $m_{2}$ only) is shown in Figure 2. It is clear that for a particular value of $K_{2}$, there are often several steady states. When nonlinear predator response functions are used, the number of steady states is also unknown a priori. The interval method provides a means to guarantee that all steady-state solutions will be found. When the simultaneous approach was used, and a single $7 \times 7$ equation system solved, the $\mathrm{CPU}$ time required for each value of $K_{2}$ averaged about 60 seconds (3.2 GHz Pentium 4). When the sequential approach was used, and a sequence of many smaller systems solved, the CPU time required for each value of $K_{2}$ averaged about 0.02 seconds. Clearly it is much more effective to use a sequential strategy. For further discussion of this problem and an interpretation of the results, see Gwaltney [18].

\subsection{Remarks}

In computing the equilibrium states in nonlinear food web models, it is possible to have a very large number of solutions. For example, Gwaltney [18] also considered a food web with 12 species and explicit resource dynamics (4 nutrients). For some 
sets of parameter values, well over 300 steady-state solutions were found by using the sequential approach with an interval-Newton method. In cases for which a large number of solutions is possible, and the number of solutions is not known, the use of interval methods for nonlinear equation solving is an attractive approach for ensuring that no solutions will be missed.

\section{Concluding Remarks}

In the examples presented here, we have shown that an interval method for nonlinear equation solving, in particular an approach incorporating the interval-Newton method, is a powerful approach for the solution of systems of nonlinear equation systems. The method provides a mathematical and computational guarantee that all solutions within a specified initial interval are enclosed. Continuing improvements in solution methods, together with advances in software and hardware for the use of intervals, will make this an increasingly attractive problem solving tool.

The validation provided by the interval approach comes at the expense of additional computation time. Essentially one has a choice between fast methods that may give an incorrect or incomplete answer, or a slower method that is guaranteed to give the correct results. Thus, a modeler may need to consider the trade off between the additional computing time and the risk of getting the wrong answer to a problem. Certainly, for "mission critical" situations, the additional computing expense is well spent. 


\section{Acknowledgements}

This work was supported in part by the Department of Education Graduate Assistance in Areas of National Needs (GAANN) Program under Grant \#P200A010448, by the donors of The Petroleum Research Fund, administered by the ACS, under Grant 35979-AC9, by the State of Indiana 21st Century Research and Technology Fund under Grant \#909010455, and by the National Oceanic and Atmospheric Administration under Grant \#NA050AR4601153.

\section{References}

[1] C. S. Adjiman, I. P. Androulakis, C. A. Floudas, A global optimization method, $\alpha \mathrm{BB}$, for general twice-differentiable constrained NLPs - II. Implementation and computational results, Computers \& Chemical Engineering 22 (1998) 1159-1179.

[2] C. S. Adjiman, S. Dallwig, C. A. Floudas, A. Neumaier, A global optimization method, $\alpha \mathrm{BB}$, for general twice-differentiable constrained NLPs - I. Theoretical advances, Computers \& Chemical Engineering 22 (1998) 1137-1158.

[3] G. Alefeld, Interval arithmetic tools for range approximation and inclusion of zeros, In: H. Bulgak, C. Zenger (Eds.), Error Control and Adaptivity in Scientific Computing, Kluwer Academic Publishers, Dordrecht, The Netherlands, 1999, pp. $1-21$. 
[4] G. Alefeld, J. Herzberger, Introduction to Interval Computations, Academic Press, Inc., New York, NY, 1983.

[5] J. Baker, An algorithm for the location of transition-states, Journal of Computational Chemistry 7 (1986) 385-395.

[6] A. Bargiela, W. Pedrycz, Granular Computing: An Introduction, Kluwer Academic Publishers, Norwell, MA, 2003.

[7] C. H. Bischof, B. Lang, W. Marquardt, M. Mönnigmann, Verified determination of singularities in chemical processes, Presented at: SCAN 2000, 9th GAMMIMACS International Symposium on Scientific Computing, Computer Arithmetic and Validated Numerics, Karlsruhe, Germany, September 18-22, 2000.

[8] G. I. Burgos-Solórzano, J. F. Brennecke, M. A. Stadtherr, Validated computing approach for high-pressure chemical and multiphase equilibrium, Fluid Phase Equilibria 219 (2004) 245-255.

[9] N. S. Dimitrova, S. M. Markov, On validated Newton type method for nonlinear equations, Interval Computations 1994(2) (1994) 27-51.

[10] Y. G. Dolgov, Developing interval global optimization algorithms on the basis of branch-and-bound and constraint propagation methods, Reliable Computing 11 (2005) 343-358. 
[11] C.-Y. Gau, J. F. Brennecke, M. A. Stadtherr, Reliable nonlinear parameter estimation in VLE modeling, Fluid Phase Equilibria 168 (2000) 1-18.

[12] C.-Y. Gau, M. A. Stadtherr, Reliable nonlinear parameter estimation using interval analysis: Error-in-variable approach, Computers \& Chemical Engineering 24 (2000) 631-637.

[13] C.-Y. Gau, M. A. Stadtherr, Dynamic load balancing for parallel interval-Newton using message passing, Computers \& Chemical Engineering 26 (2002) 811-825.

[14] C.-Y. Gau, M. A. Stadtherr, New interval methodologies for reliable chemical process modeling, Computers \& Chemical Engineering 26 (2002) 827-840.

[15] V. Gehrke, W. Marquardt, A singularity theory approach to the study of reactive distillation, Computers \& Chemical Engineering 21 (1997) S1001-S1006.

[16] L. Granvilliers, On the combination of interval constraint solvers, Reliable Computing 7 (2001) 467-483.

[17] L. Granvilliers, F. Benhamou, Algorithm 852: RealPaver: An interval solver using constraint satisfaction techniques, ACM Transactions on Mathematical Software 32 (2006) 138-156.

[18] C. R. Gwaltney, Reliable Location Of Equlibrium States And Bifurcations In Nonlinear Dynamical Systems With Applications In Food Web Modeling And 
Chemical Engineering, PhD thesis, University of Notre Dame, Notre Dame, IN, 2006.

[19] C. R. Gwaltney, M. A. Stadtherr, Reliable computation of equilibrium states and bifurcations in nonlinear dynamics, Lecture Notes in Computer Science 3732 (2006) 122-131.

[20] C. R. Gwaltney, M. P. Styczynski, M. A. Stadtherr, Reliable computation of equilibrium states and bifurcations in food chain models, Computers \& Chemical Engineering 28 (2004) 1981-1996.

[21] E. Hansen, Sharpening interval computations, Reliable Computing 12 (2006) $21-34$.

[22] E. Hansen, S. Sengupta, Bounding solutions of systems of equations using interval-analysis, BIT 21 (1981) 203-211.

[23] E. R. Hansen, R. I. Greenberg, An interval Newton method, Applied Mathematics \& Computation 12 (1983) 89-98.

[24] E. R. Hansen, G. W. Walster, Global Optimization Using Interval Analysis, Marcel Dekker, New York, NY, 2004.

[25] R. A. Heidemann, J. M. Mandhane, Some properties of the NRTL equation in correlating liquid-liquid equilibrium data, Chemical Engineering Science 28 (1973) 1213-1221. 
[26] S. Herbort, D. Ratz, Improving the efficiency of a nonlinear-system-solver using a componentwise newton method, Bericht 2/1997, Institut für Angewandte Mathematik, Universität Karlsruhe (TH), Karlsruhe, Germany, 1997.

[27] J. Z. Hua, J. F. Brennecke, M. A. Stadtherr, Reliable phase stability analysis for cubic equation of state models, Computers \& Chemical Engineering 20 (1996) S395-S400.

[28] J. Z. Hua, J. F. Brennecke, M. A. Stadtherr, Reliable prediction of phase stability using an interval-Newton method, Fluid Phase Equilibria 116 (1996) 52-59.

[29] J. Z. Hua, J. F. Brennecke, M. A. Stadtherr, Enhanced interval analysis for phase stability: Cubic equation of state models, Industrial \& Engineering Chemistry Research, 37 (1998), pp. 1519-1527.

[30] J. Z. Hua, J. F. Brennecke, M. A. Stadtherr, Reliable computation of phase stability using interval analysis: Cubic equation of state models, Computers \& Chemical Engineering 22 (1998) 1207-1214.

[31] J. Z. Hua, R. W. Maier, S. R. Tessier, J. F. Brennecke, M. A. Stadtherr, Interval analysis for thermodynamic calculations in process design: A novel and completely reliable approach, Fluid Phase Equilibria 158 (1999) 607-615.

[32] ICOS, See http://www.essi.fr/verb| |lebbah/icos. 
[33] J. Jacq, L. Asselineau, Binary liquid-liquid equilibria. Multiple solutions for the NRTL equation, Fluid Phase Equilibria 14 (1983) 185-192.

[34] L. Jaulin, M. Kieffer, O. Didrit, É. Walter, Applied Interval Analysis, SpringerVerlag, London, 2001.

[35] R. L. June, A. T. Bell, D. N. Theodorou, Transition-state studies of xenon and $\mathrm{SF}_{6}$ diffusion in silicalite, Journal of Physical Chemistry 95 (1991) 8866-8878.

[36] J. Karger, D. M. Ruthven, Diffusion in Zeolites and Other Microporous Solids, Wiley, New York, NY, 1992.

[37] R. B. Kearfott, Preconditioners for the interval Gauss-Seidel method, SIAM Journal on Numerical Analysis 27 (1990) 804-822.

[38] R. B. Kearfott, Algorithm 763; INTERVAL_ARITHMETIC: A Fortran-90 module for an interval data type, ACM Transactions on Mathematical Software 22 (1996) 385-392.

[39] R. B. Kearfott, Interval computations: Introduction, uses, and resources, Euromath Bulletin 2 (1996) 95-112.

[40] R. B. Kearfott, Rigorous Global Search: Continuous Problems, Kluwer Academic Publishers, Dordrecht, The Netherlands, 1996.

[41] R. B. Kearfott, Validated constraint solving - Practicalities, pitfalls, and new developments, Reliable Computing 11 (2005) 383-391. 
[42] R. B. Kearfott, M. Dawande, K. Du, C. Hu, INTLIB: A portable Fortran-77 elementary function library, Interval Computations 1992(3) (1992) 96-105.

[43] R. B. Kearfott, M. Dawande, K. Du, C. Hu, Algorithm 737: INTLIB: A portable Fortran-77 elementary function library, ACM Transactions on Mathematical Software 20 (1994) 447-459.

[44] R. B. Kearfott, C. Hu, M. Novoa III, A review of preconditioners for the interval Gauss-Seidel method, Interval Computations 1991(1) (1991) 59-85.

[45] R. B. Kearfott, M. Novoa III, Algorithm 681: INTBIS, A portable interval Newton/bisection package, ACM Transactions on Mathematical Software 16 (1990) $152-157$.

[46] L. V. Kolev, A new method for global solution of systems of non-linear equations, Reliable Computing 4 (1998) 125-146.

[47] L. V. Kolev, An improved method for global solution of non-linear systems, Reliable Computing 5 (1999) 103-111.

[48] V. Kreinovich, A. Bernat, Parallel algorithms for interval computations: An introduction, Interval Computations 1994(3) (1994) 6-62.

[49] C. Lavor, A deterministic approach for global minimization of molecular potential energy functions, International Journal of Quantum Chemistry 95 (2003) 336343. 
[50] M. Lerch, G. Tischler, J. Wolff von Gudenberg, W. Hofschuster, W. Krämer, FILIB ++ , A fast interval library supporting containment computations, ACM Transactions on Mathematical Software 32 (2006) 299-324.

[51] Y. Lin, M. A. Stadtherr, Advances in interval methods for deterministic global optimization in chemical engineering, Journal of Global Optimization 29 (2004) $281-296$.

[52] Y. Lin, M. A. Stadtherr, Locating stationary points of sorbate-zeolite potential energy surfaces using interval analysis, Journal of Chemical Physics 121 (2004) $10159-10166$.

[53] Y. Lin, M. A. Stadtherr, LP strategy for the interval-Newton method in deterministic global optimization, Industrial \& Engineering Chemistry Research 43 (2004) 3741-2749.

[54] Y. Lin, M. A. Stadtherr, Deterministic global optimization of molecular structures using interval analysis, Journal of Computational Chemistry 26 (2005) $1413-1420$.

[55] R. W. Maier, J. F. Brennecke, M. A. Stadtherr, Reliable computation of homogeneous azeotropes, AIChE Journal 44 (1998) 1745-1755.

[56] R. W. Maier, J. F. Brennecke, M. A. Stadtherr, Reliable computation of reactive azeotropes, Computers \& Chemical Engineering 24 (2000) 1851-1858. 
[57] R. W. Maier, M. A. Stadtherr, Reliable density-functional-theory calculations of adsorption in nanoscale pores, AIChE Journal 47 (2001) 1874-1884.

[58] K. Makino, M. Berz, Efficient control of the dependency problem based on Taylor model methods, Reliable Computing 5 (1999) 3-12.

[59] A. C. Mattelin, L. A. J. Verhoeye, The correlation of binary miscibility data by means of the NRTL equation, Chemical Engineering Science 30 (1975) 193-200.

[60] K. I. M. McKinnon, C. G. Millar, M. Mongeau, Global optimization for the chemical and phase equilibrium problem using interval analysis, In: C. A. Floudas and P. M. Pardalos (Eds.), State of the Art in Global Optimization Computational Methods and Applications, Kluwer Academic Publishers, Dordrecht, The Netherlands, 1996.

[61] K. Min, L. Qi, S. Zuhe, On the componentwise Krawczyk-Moore iteration, Reliable Computing 5 (1999) 359-370.

[62] M. Mönnigmann, W. Marquardt, Normal vectors on manifolds of critical points for parametric robustness of equilibrium solutions of ODE systems, Journal of Nonlinear Science 12 (2002) 85-112.

[63] R. E. Moore, Interval Analysis, Prentice-Hall, Englewood Cliffs, NJ, 1966.

[64] R. E. Moore, A test for existence of solutions to nonlinear systems, SIAM Journal on Numerical Analysis 14 (1977) 611-615. 
[65] R. E. Moore, Methods and Applications of Interval Analysis, SIAM, Philadelphia, PA, 1979.

[66] V. M. Nesterov, How to use monotonicity-type information to get better estimates of the range of real-valued functions, Interval Computations 1993(4) (1993) 3-12.

[67] A. Neumaier, Interval Methods for Systems of Equations, Cambridge University Press, Cambridge, UK, 1990.

[68] A. Neumaier, Taylor forms - Use and limits, Reliable Computing 9 (2002) 43-79.

[69] D. H. Olson, G. T. Kokotailo, S. L. Lawton, W. M. Meier, Crystal structure and structure-related properties of ZSM-5, Journal of Physical Chemistry 85 (1981) 2238-2243.

[70] PROFIL/BIAS, See http://www.ti3.tu-harburg.de/Software/ PROFILEnglisch.html.

[71] J. D. Pryce, G. F. Corliss, Interval arithmetic with containment sets, Computing 78 (2006) 251-276.

[72] H. Ratschek, J. Rokne, Computer Methods for the Range of Functions, Ellis Horwood, Chichester, UK, 1984.

[73] H. Renon, J. M. Prausnitz, Local compositions in thermodynamic excess functions for liquid mixtures, AIChE Journal 14 (1968) 135-144. 
[74] S. M. Rump, Verification methods for dense and sparse systems of equations, In: J. Herzberger (Ed.), Topics in Validated Computations - Studies in Computational Mathematics, Elsevier, Amsterdam, The Netherlands, 1994, pp. 63-135.

[75] S. M. Rump, INTLAB - INTerval LABoratory, In: T. Csendes (Ed.), Developments in Reliable Computing, Kluwer Academic Publishers, Dordrecht, The Netherlands, 1999, pp. 77-104.

[76] C. A. Schnepper, M. A. Stadtherr, Application of a parallel interval Newton/generalized bisection algorithm to equation-based chemical process flowsheeting, Interval Computations 1993(4) (1993) 40-64.

[77] C. A. Schnepper, M. A. Stadtherr, Robust process simulation using interval methods, Computers \& Chemical Engineering 20 (1996) 187-199.

[78] A. M. Scurto, G. Xu, J. F. Brennecke, M. A. Stadtherr, Phase behavior and reliable computation of high-pressure solid-fluid equilibrium with cosolvents, Industrial \& Engineering Chemistry Research 42 (2003) 6464-6475.

[79] S. P. Shary, Krawczyk operator revised, In: Proceedings of International Conference on Computational Mathematics ICCM-2004, 2004.

[80] L. Simcik, P. Linz, Boundary-based interval Newton's method, Interval Computations 1993(4) (1993) 89-99. 
[81] J. M. Sørensen, W. Arlt, Liquid-Liquid Equilibrium Data Collection, Chemistry Data Series, Vol. V, Parts 1-3, DECHEMA, Frankfurt/Main, Germany, 19791980.

[82] M. A. Stadtherr, C. A. Schnepper, J. F. Brennecke, Robust phase stability analysis using interval methods, AIChE Symposium Series 91(304) (1995) 356-359.

[83] B. A. Stradi, J. F. Brennecke, J. P. Kohn, M. A. Stadtherr, Reliable computation of mixture critical points, AIChE Journal 47 (2001) 212-221.

[84] S. R. Tessier, J. F. Brennecke, M. A. Stadtherr, Reliable phase stability analysis for excess Gibbs energy models, Chemical Engineering Science 55 (2000) 17851796.

[85] C. J. Tsai, K. D. Jordan, Use of an eigenmode method to locate the stationarypoints on the potential-energy surfaces of selected argon and water clusters, Journal of Physical Chemistry 97 (1993) 11227-11237.

[86] P. van Hentenryck, L. Michel, Y. Deville, Numerica: A Modeling Language for Global Optimization, The MIT Press, Cambridge, MA, 1997.

[87] K. M. Westerberg, C. A. Floudas, Locating all transition states and studying the reaction pathways of potential energy surfaces, Journal of Chemical Physics 110 (1999) 9259-9295. 
[88] G. Xu, J. F. Brennecke, M. A. Stadtherr, Reliable computation of phase stability and equilibrium from the SAFT equation of state, Industrial \& Engineering Chemistry Research 41 (2002) 938-952.

[89] G. Xu, W. D. Haynes, M. A. Stadtherr, Reliable phase stability analysis for asymmetric models, Fluid Phase Equilibria 235 (2005) 152-165.

[90] G. Xu, A. M. Scurto, M. Castier, J. F. Brennecke, M. A. Stadtherr, Reliable computational of high-pressure solid-fluid equilibrium, Industrial \& Engineering Chemistry Research 39 (2000) 1624-1636.

[91] K. Yamamura, Finding all solutions of nonlinear equations using linear combinations of functions, Reliable Computing 6 (2000) 105-113. 
Table 1: Comparison of NRTL parameter estimates for the mixture n-butanol and water $(\alpha=0.4, T=363 \mathrm{~K})$ obtained by Heidemann and Mandhane [25] and by the use of an interval method.

\begin{tabular}{|c|c|c|c|c|}
\hline & \multicolumn{2}{|c|}{ Reference [25] } & \multicolumn{2}{|c|}{ Interval Method } \\
\hline Solution & $\tau_{12}$ & $\tau_{21}$ & $\tau_{12}$ & $\tau_{21}$ \\
\hline 1 & 0.0075 & 3.8021 & 0.0075 & 3.8021 \\
\hline 2 & 10.182 & 3.8034 & 10.178 & 3.8034 \\
\hline 3 & -73.824 & -15.822 & & \\
\hline
\end{tabular}


Table 2: NRTL parameter estimates for the mixture 1,4-dioxane and 1,2,3propanetriol $(\alpha=0.15$ and $T=298.15 \mathrm{~K})$ found using an interval method. Mattelin and Verhoeye [59] reported finding 6 solutions.

\begin{tabular}{cll} 
& \multicolumn{2}{l}{ Interval Method } \\
\hline Solution & $\tau_{12}$ & $\tau_{21}$ \\
\hline 1 & 5.6379 & -0.59940 \\
& & \\
2 & 13.478 & -82.941 \\
& & \\
3 & 38.642 & 13.554 \\
& & \\
4 & 39.840 & 3.0285 \\
\hline
\end{tabular}


Table 3: Stationary points of the potential energy surface of xenon in silicalite

\begin{tabular}{|c|c|c|c|c|c|}
\hline No. & Type & Energy $(\mathrm{kcal} / \mathrm{mol})$ & $\mathrm{x}(\AA)$ & $\mathrm{y}(\AA)$ & $z(\AA)$ \\
\hline 1 & minimum & -5.9560 & 3.9956 & 4.9800 & 12.1340 \\
\hline 2 & minimum & -5.8763 & 0.3613 & 0.9260 & 6.1112 \\
\hline 3 & minimum & -5.8422 & 5.8529 & 4.9800 & 10.8790 \\
\hline 4 & minimum & -5.7455 & 1.4356 & 4.9800 & 11.5540 \\
\hline 5 & minimum & -5.1109 & 0.4642 & 4.9800 & 6.0635 \\
\hline 6 & 1st order & -5.7738 & 5.0486 & 4.9800 & 11.3210 \\
\hline 7 & 1st order & -5.6955 & 0.0000 & 0.0000 & 6.7100 \\
\hline 8 & 1st order & -5.6060 & 2.3433 & 4.9800 & 11.4980 \\
\hline 9 & 1st order & -4.7494 & 0.1454 & 3.7957 & 6.4452 \\
\hline 10 & 1st order & -4.3057 & 9.2165 & 4.9800 & 11.0110 \\
\hline 11 & 1st order & -4.2380 & 0.0477 & 3.9147 & 8.3865 \\
\hline 12 & 1st order & -4.2261 & 8.6361 & 4.9800 & 12.8560 \\
\hline 13 & 1st order & -4.1405 & 0.5925 & 4.9800 & 8.0122 \\
\hline 14 & 2nd order & -4.1404 & 0.5883 & 4.8777 & 8.0138 \\
\hline 15 & 2nd order & -4.1027 & 9.1881 & 4.1629 & 11.8720 \\
\hline
\end{tabular}




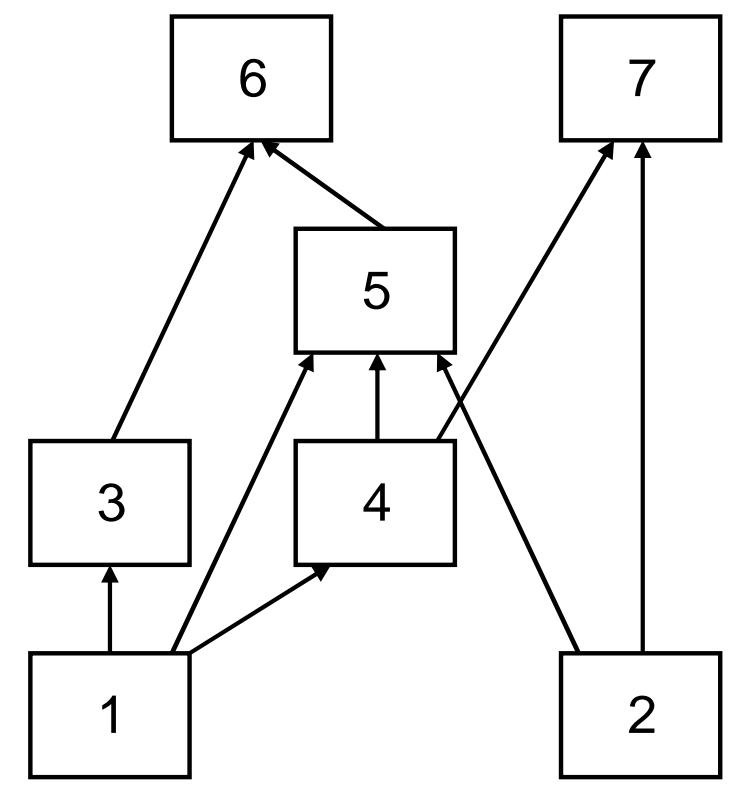

Figure 1: Diagram illustrating the predation relationships in the seven species food web model. 

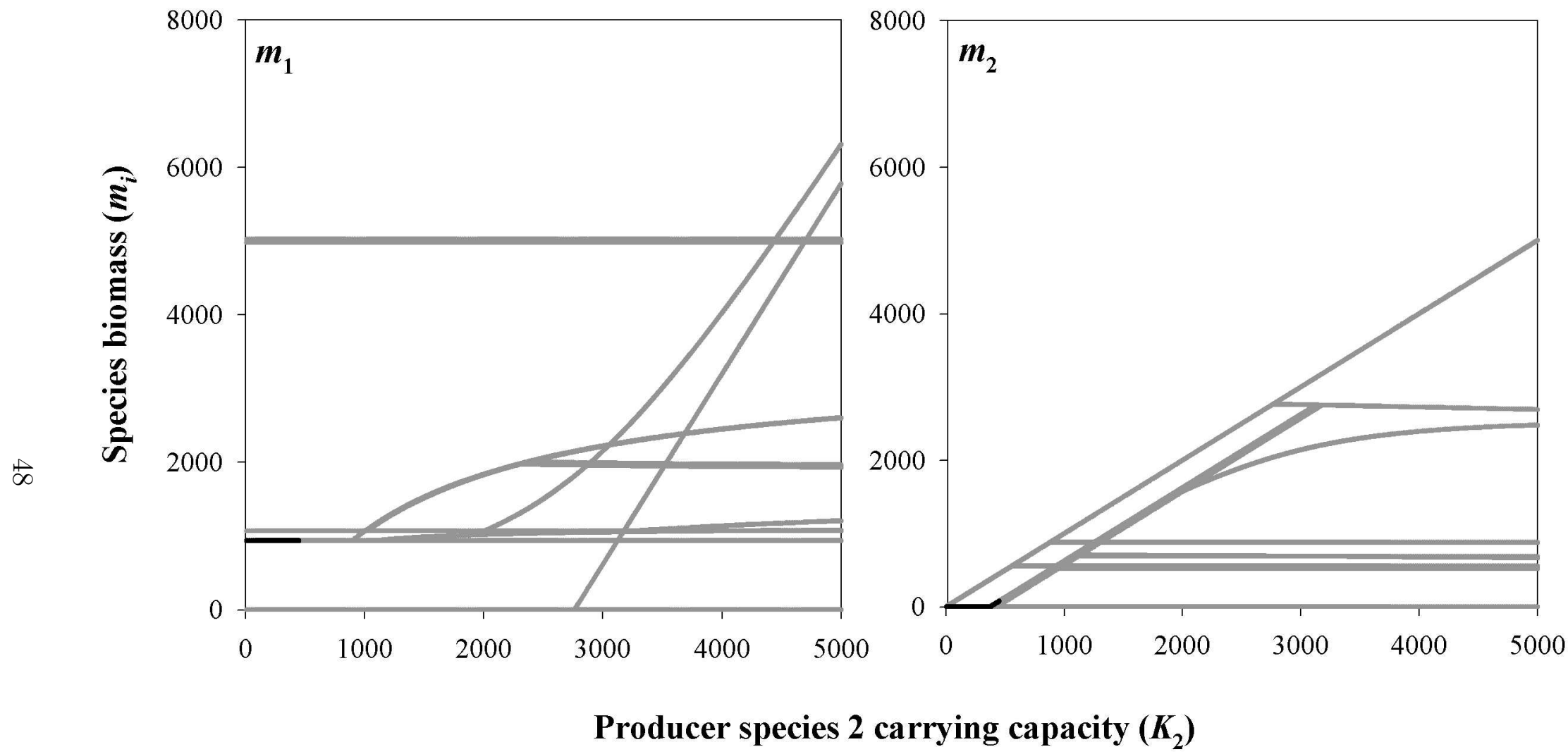

Figure 2: Solution branch diagrams illustrating the change in the steady-state biomass values of species $1\left(m_{1}\right)$ and species $2\left(m_{2}\right)$ with change in species 2 carrying capacity $\left(K_{2}\right)$ for the seven-species food web model. Black lines indicate stable equilibria. Gray lines indicate unstable equilibria. 


\section{Keywords}

Interval analysis; Equation solving; Interval-Newton method; Phase equilibrium; Transition state analysis; Food webs

\section{Biographical Sketch}

Mark A. Stadtherr is Professor of Chemical and Biomolecular Engineering at the University of Notre Dame. He has a B.Ch.E. Degree from the University of Minnesota and a Ph.D. Degree from the University of Wisconsin. He was awarded the 1998 Computing in Chemical Engineering Award by the American Institute of Chemical Engineers. His research interests include the application of interval methods to global optimization, nonlinear algebraic equation solving, and systems of ordinary differential equations. 